\title{
Associations of myocardial bridging with adverse cardiac events: a meta-analysis of published observational cohort studies involving 4,556 individuals
}

\author{
Changsheng Zhu, Shuiyun Wang, Hao Cui, Bing Tang, Shengwei Wang \\ Department of Cardiovascular Surgery, Fuwai Hospital, National Center for Cardiovascular Diseases, Chinese Academy of Medical Sciences and \\ Peking Union Medical College, Beijing 100037, China \\ Contributions: (I) Conception and design: C Zhu, S Wang; (II) Administrative support: S Wang; (III) Provision of study materials or patients: C Zhu, \\ S Wang, B Tang; (IV) Collection and assembly of data: C Zhu, S Wang, H Cui, B Tang; (V) Data analysis and interpretation: C Zhu, S Wang, H Cui; \\ (VI) Manuscript writing: All authors; (VII) Final approval of manuscript: All authors. \\ Correspondence to: Shuiyun Wang, MD. Department of Cardiovascular Surgery, Fuwai Hospital, National Center for Cardiovascular Diseases, \\ Chinese Academy of Medical Sciences and Peking Union Medical College, Beilishi Road 167, Xicheng District, Beijing 100037, China. \\ Email: wsymd@sina.com.
}

\begin{abstract}
Background: Data derived from small series have demonstrated an association of myocardial bridge (MB) with adverse cardiac events, while $\mathrm{MB}$ has been traditionally considered as a benign condition. Hence, the precise clinical implications of $\mathrm{MB}$ on prognosis remains inconsistent. Our purpose is to perform a metaanalysis to assess the clinical implications of $\mathrm{MB}$ on prognosis.

Methods: We performed an extensive search of PubMed and reference lists of relevant articles. Studies which compared prognosis between subjects with and without MB were identified from 1960 to 31 March 2018. Studies selection was limited to human data and restricted to English language.

Results: Six eligible studies were included in current meta-analysis. Of 4,556 subjects, 1,389 (30.5\%) presented MB. MB was associated with an increased risk of adverse cardiac events [odds ratio (OR), 1.71; 95\% confidence interval (CI): 1.29 to 2.26; $\mathrm{P}=0.0002$ ], non-fatal myocardial infarction (OR: 3.17; 95\% CI: 1.21 to $8.31 ; \mathrm{P}=0.02$ ), and angina requiring hospitalization (OR: $2.31 ; 95 \% \mathrm{CI}: 1.55$ to 3.45 ; $\mathrm{P}<0.0001$ ), respectively, compared with subjects without $\mathrm{MB}$.

Conclusions: This meta-analysis of currently available observational cohort studies suggests that MB has an association with adverse cardiac events. Further prospective multicenter studies with large sample size are needed to confirm current findings. Moreover, studies refining the impact of different types of $M B$ on cardiac events, myocardial ischemia, and symptoms requiring therapy, may provide more insights to this issue.
\end{abstract}

Keywords: Myocardial bridging (MB); adverse cardiac events (ACEs); prognosis; meta-analysis

Submitted Oct 20, 2019. Accepted for publication Jan 10, 2020.

doi: 10.21037/atm.2020.02.24

View this article at: http://dx.doi.org/10.21037/atm.2020.02.24

\section{Introduction}

Myocardial bridging (MB) is a congenital variant of coronary artery anatomy which indicates the myocardium overlying an intramural segment of an epicardial coronary artery. MB mostly involves the middle segment of the left anterior descending artery (LAD), though its prevalence varies according to different imaging modalities and methods used (1-3). The data derived from small sample studies indicate $\mathrm{MB}$ may cause a variety of adverse cardiac events (ACEs) including myocardial infarction (MI), lifethreatening arrhythmias, and sudden cardiac death (3-6). In this regard, the clinical relevance of $\mathrm{MB}$ is of crucial 
importance. Actually, $\mathrm{MB}$ has long been considered as a benign condition given that the prevalence of $\mathrm{MB}$ is usually high in autopsy and blood flow runs through normal coronary artery mainly during diastolic phase, while $\mathrm{MB}$ compression occurs during systolic phase and only approximately in one third of subjects with $\mathrm{MB}$ (3,7-10). Therefore, the precise clinical implication of $\mathrm{MB}$ on prognosis remains controversial. We aimed to conduct a meta-analysis of currently available evidence to examine the clinical implication of $\mathrm{MB}$ on prognosis among general population.

\section{Methods}

The present meta-analysis was performed with a predefined protocol and complied with PRISMA and MOOSE guidelines (Table S1,S4).

\section{Search strategy}

An extensive search of PubMed with English language restriction was performed using the terms like "myocardial bridging", "myocardial bridge", "intramural coronary artery", "mural coronary artery", "coronary artery overbridging", "tunneled artery" and "myocardial loop". Additional reference lists of relevant articles were reviewed. Studies published between 1960 in which year MB was first reported angiographically and 31 March 2018 were identified $(3,4,11)$. The detailed search strategy was presented in Table $S 2$.

\section{Study selection}

We only included observational cohort studies either prospective or retrospective comparing the outcome of subjects with and without $\mathrm{MB}$, which represent the best level of clinical evidence to date. Inclusion criteria were the followings: (I) population referred consecutively to hospital for imaging examination of coronary artery; (II) explicit description of inclusion or exclusion criteria; and (III) comparison of outcome during follow-up between subjects with and without MB. Exclusion criteria were the followings: (I) studies incapable of extracting specific data; (II) studies dealing with patient population with specific disease like hypertrophic cardiomyopathy. Potentially eligible studies were evaluated by two independent reviewers (C Zhu and S Wang) as well as data extraction and quality evaluation of the final included studies. Any discrepancies were resolved by consensus meeting of all authors of this meta-analysis subsequently.

\section{Quality evaluation of included studies}

The Newcastle-Ottawa Scale for cohort studies, which is a "star system" providing an easy and convenient quality assessment of nonrandomized studies in a systematic review, was used to evaluate the quality of included studies on three perspectives: selection of cohorts, comparability of cohorts, and ascertainment of outcome for cohorts $(12,13)$. Nine stars represent the highest study quality. At least 5 stars were defined to be adequate quality for inclusion in the present meta-analysis. With regard to evaluation for publication bias of included studies, the visualized funnel plot was used if applicable.

\section{Outcomes}

The primary outcome was defined as ACEs including cardiovascular death and non-fatal MI. Secondary outcomes were non-fatal MI, angina requiring hospitalization, and all-cause mortality. Furthermore, a composite endpoint was defined as a combination of ACEs, non-cardiac death and angina requiring hospitalization.

\section{Statistical analysis}

Analysis was conducted using Review Manager Version 5.3 (The Cochrane Collaboration, Update Software, Copenhagen, The Nordic Cochrane Centre). Heterogeneity test was measured utilizing the $\mathrm{v}^{2}$ test (Cochrane's $\mathrm{Q}$ ) and $\mathrm{I}^{2}$ value. $\mathrm{I}^{2}$ values less than $50 \%, 50 \%$ to $75 \%$, and more than $75 \%$ represent a low, moderate, high degree of heterogeneity, respectively. If homogenous, fixed-effect model was used. Otherwise, a random-effects model was used. Odds ratio (OR) was calculated for dichotomous variables with $95 \%$ confidence interval (CI). An OR represents the ratio between odds of outcomes in the context of a particular exposure and odds of outcomes in absence of the exposure. A P value of less than 0.05 was considered statistically significant.

\section{Results}

\section{Selection of studies}

Six observational cohort studies were included in the 


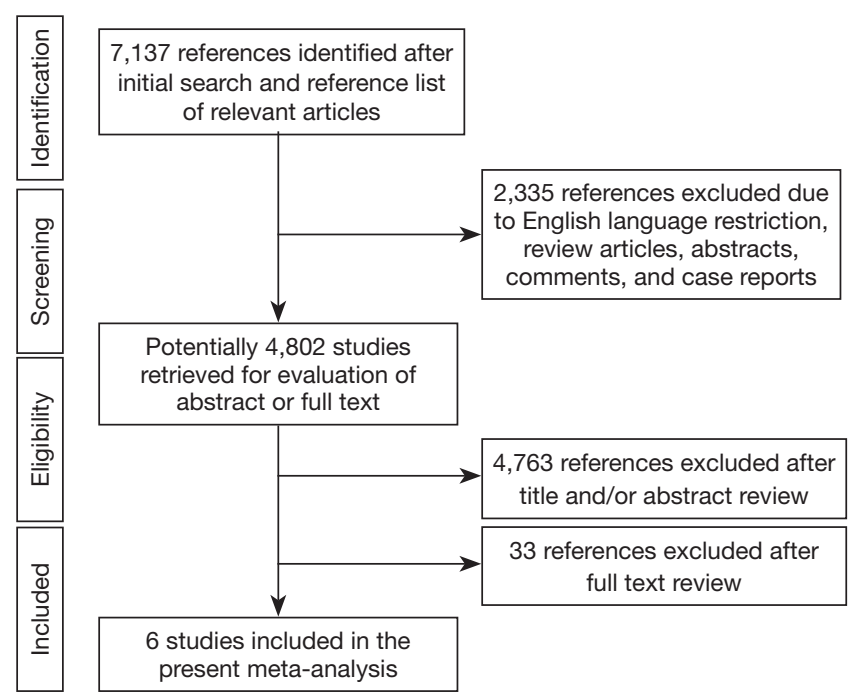

Figure 1 Flow diagram of search strategy and study selection in the present meta-analysis.

present meta-analysis for data extraction which yielded a total of 4,556 selected subjects (Figure 1) (11,14-18). Of these six included studies, only the study by Rubinshtein et al. was prospective, whereas the remaining 5 studies were retrospective. The study by Rubinshtein et al. included subjects with compromised left ventricular function or valvular heart disease who were referred to rule out obstructive coronary artery disease (11). In contrast, the study by Kim et al. excluded subjects with any risk factors of chest pain including valvular heart disease (18). The detailed inclusion and exclusion criteria and outcome measurements of selected studies were presented in Table 1. Besides, the study by Kim et al. assessed MB with coronary angiography. Table 2 demonstrates data extracted from all included studies in the present meta-analysis. All subjects were in absence of prior coronary heart disease or obstructive coronary artery disease which was defined as equal to or more than $50 \%$ coronary luminal stenosis of any coronary artery.

The quality evaluation of selected studies was demonstrated in Table S3. None of these six included studies provided information on losses to follow-up.

\section{Pooled prevalence and characteristics of $M B$}

Of the 4,556 selected subjects included, 1,389 had MB. Thus, the pooled prevalence of $\mathrm{MB}$ in the present study is $30.5 \%$. Most MB involved the LAD, which was consistent among included studies. Three studies reported MB with

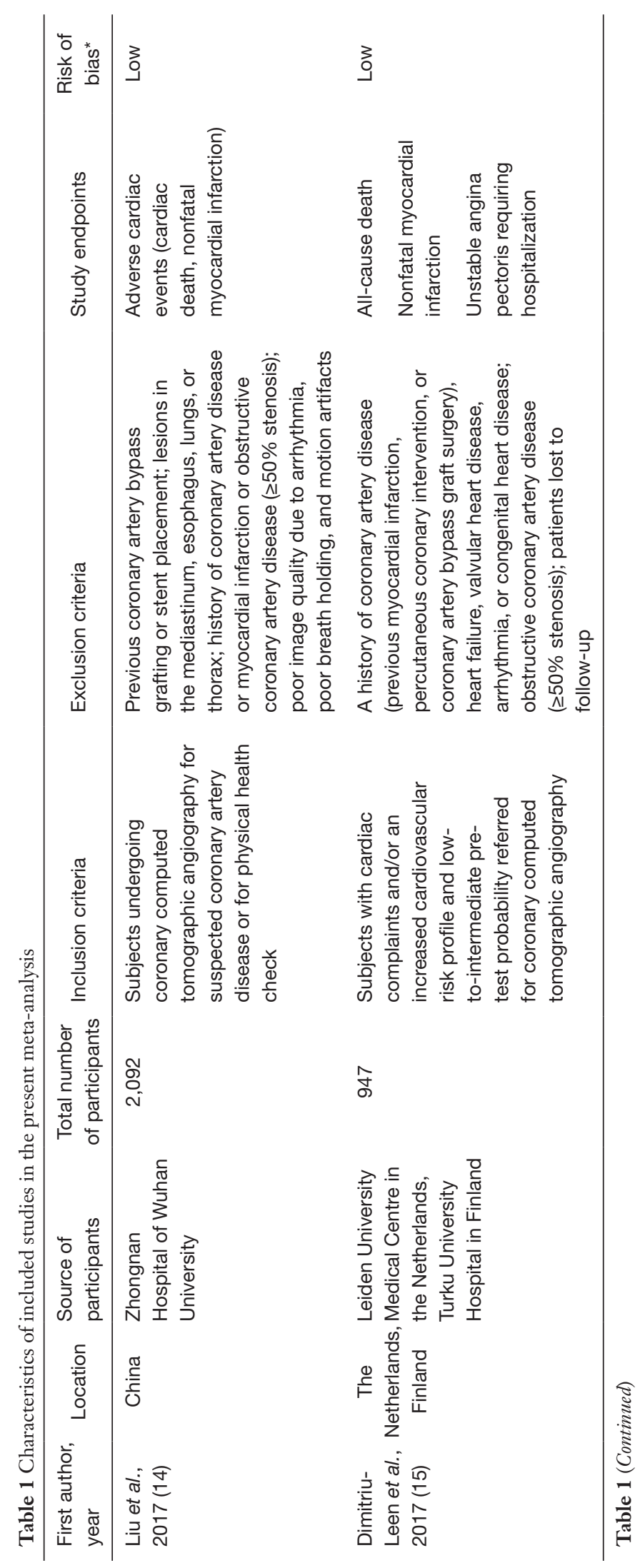




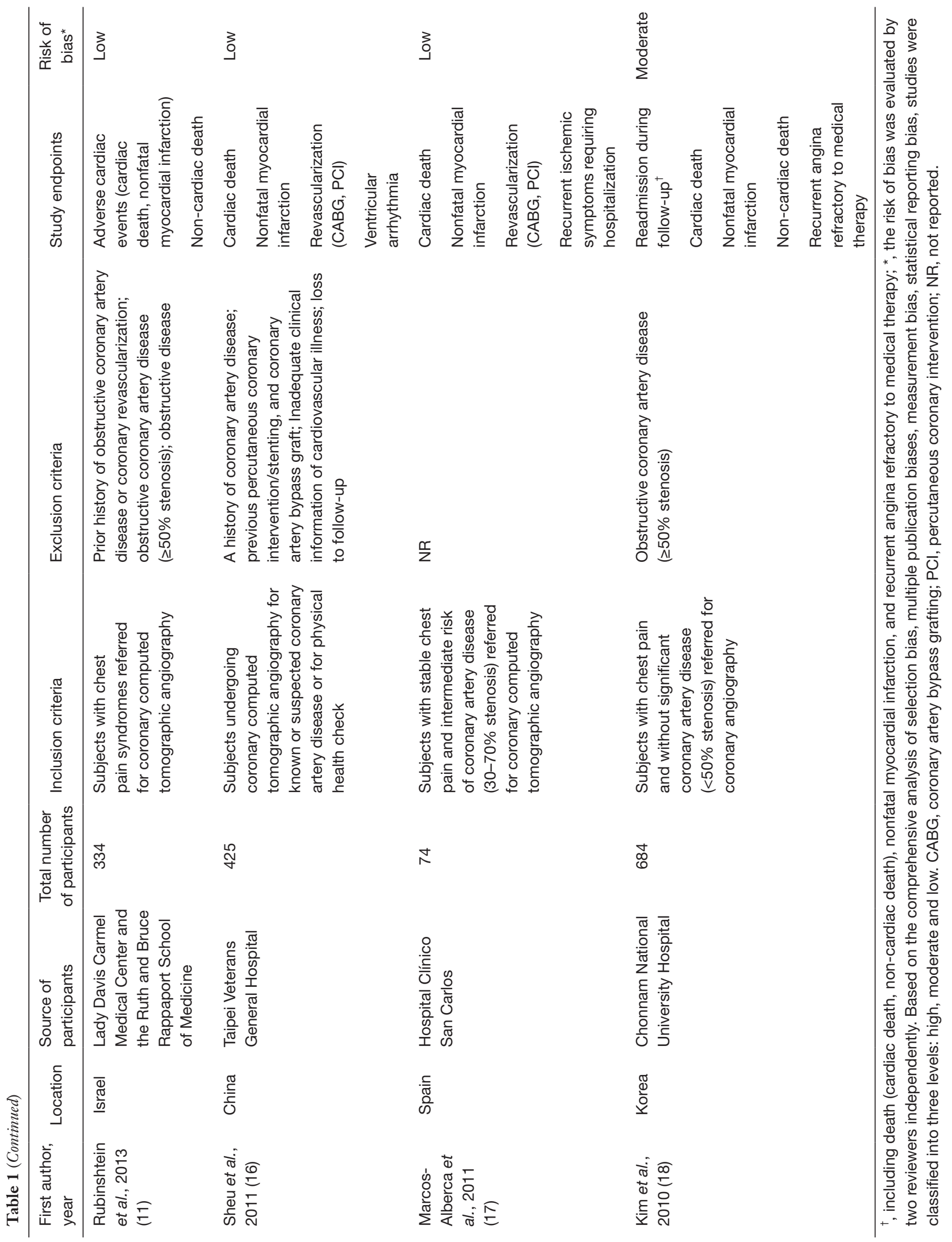




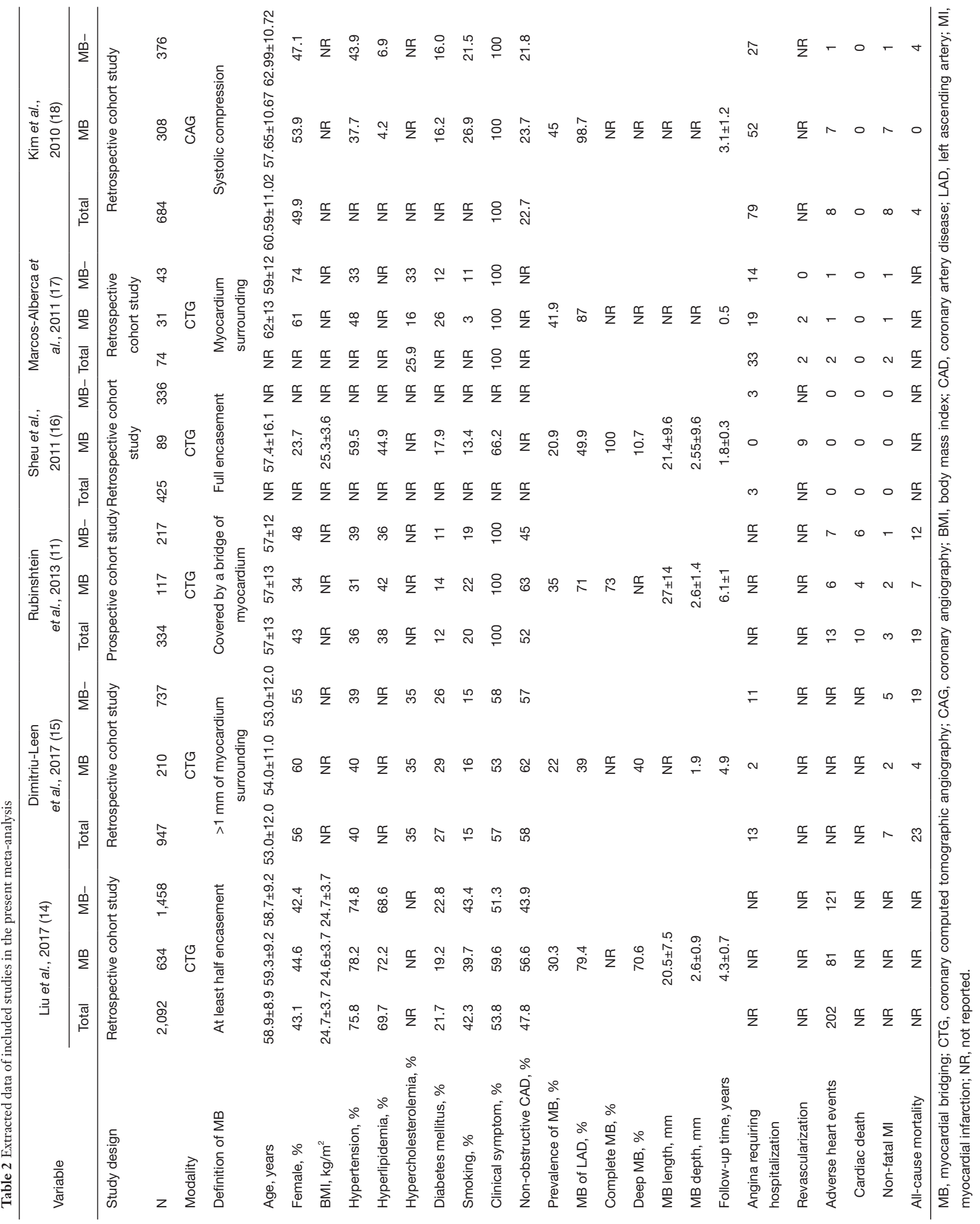


A

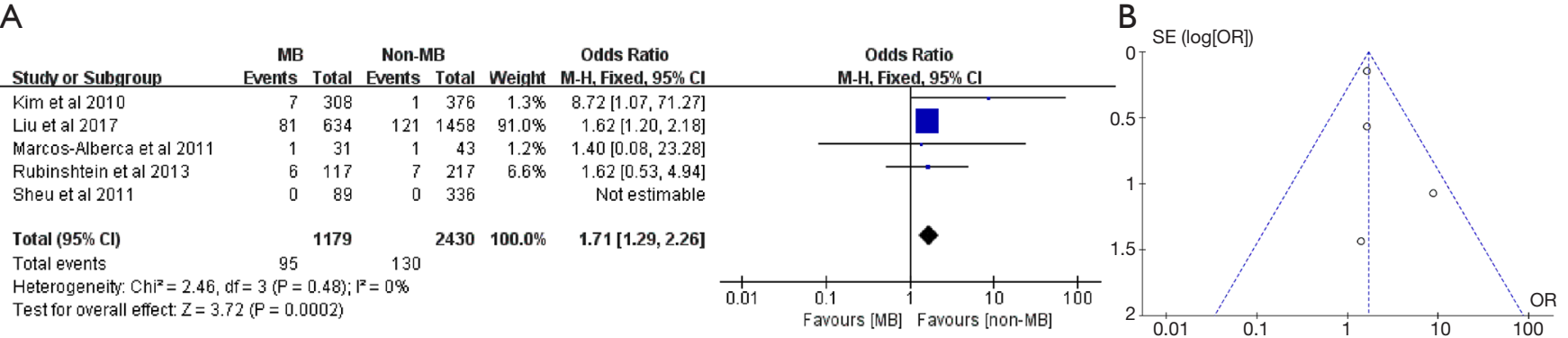

Figure 2 Pooled risk of adverse cardiac events. (A) Forest plot of included studies describing adverse cardiac events during follow-up. Subjects with myocardial bridging had higher risk of experiencing adverse cardiac events; (B) corresponding funnel plot of included studies. MB, myocardial bridge; CI, confidence interval; OR, odds ratio; SE, standard error.

A
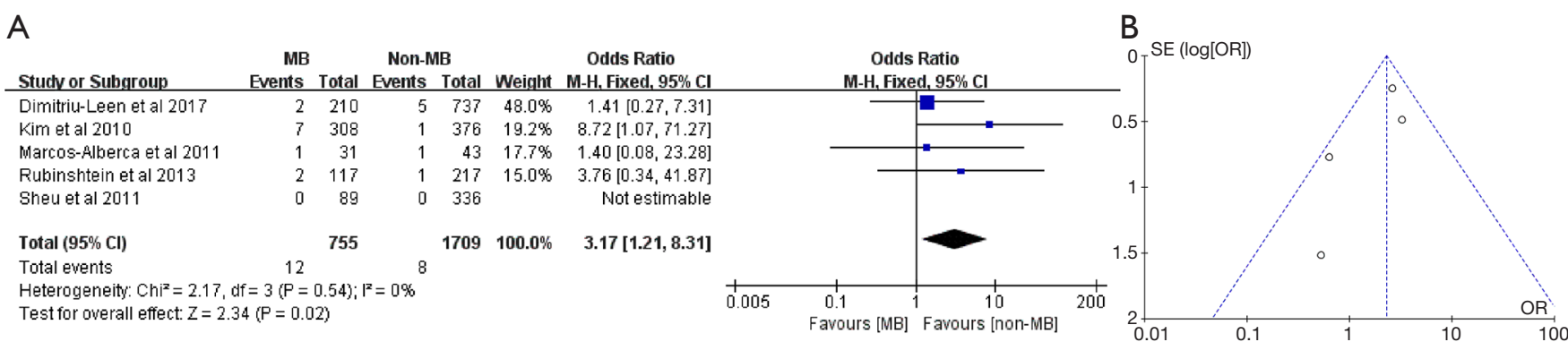

Figure 3 Pooled risk of non-fatal myocardial infarction. (A) Forest plot of included studies describing non-fatal myocardial infarction during follow-up. Subjects with myocardial bridging had higher risk of experiencing non-fatal myocardial infarction; (B) corresponding funnel plot of included studies. MB, myocardial bridge; CI, confidence interval; OR, odds ratio; SE, standard error.

mean length of 2 to $3 \mathrm{~mm}$ and mean depth of $2.6 \mathrm{~mm}$ $(11,14,16)$.

\section{Primary outcome}

ACEs were reported in five included studies comprising a total of 225 events among 3,609 subjects $(11,14,16-18)$. The pooled incidences of ACEs were $8.1 \%$ and $5.3 \%$ in subjects with $\mathrm{MB}$ and without $\mathrm{MB}$, respectively. On pooled analysis, subjects with $\mathrm{MB}$ had an increased risk of ACEs compared with subjects without MB (OR: 1.71; 95\% CI: 1.29 to 2.26, $\mathrm{P}=0.0002$ ) (Figure 2A). There was no statistical significance of heterogeneity test between included studies (Cochrane Q $\left.=2.46, \mathrm{P}=0.48, \mathrm{I}^{2}=0 \%\right)$. Besides, the corresponding funnel plot indicated that no publication bias existed (Figure $2 B$ ).

Sensitivity analysis was performed by only including five studies which used coronary computed tomographic angiography for detection of MB. Results were unchanged for ACEs in subjects with $\mathrm{MB}$ compared to that in subjects without MB (OR: 1.62; 95\% CI: 1.21 to $2.15, \mathrm{P}=0.001$ )
(Figure S1).

\section{Secondary outcomes}

Non-fatal MI was reported in five studies comprising a total of 20 events among 2,464 subjects $(11,15-18)$. The pooled incidences of non-fatal MI were $1.6 \%$ and $0.5 \%$ in subjects with $\mathrm{MB}$ and without $\mathrm{MB}$, respectively. Subjects with $\mathrm{MB}$ had an increased risk of experiencing non-fatal MI compared with subjects without MB (OR: 3.17; 95\% CI: 1.21 to $8.31, \mathrm{P}=0.02$ ) (Figure 3). There was no statistical heterogeneity for the outcome of non-fatal MI between included studies (Cochrane $\mathrm{Q}=2.17, \mathrm{P}=0.54, \mathrm{I}^{2}=0 \%$ ).

Angina requiring hospitalization was reported in 4 studies comprising a total of 128 events among 2,130 subjects (15-18). The pooled incidences of angina requiring hospitalization were $11.4 \%$ and $3.7 \%$ in subjects with $\mathrm{MB}$ and without $M B$, respectively. Subjects with $M B$ had an increased risk of angina requiring hospitalization compared with subjects without MB (OR: 2.31; 95\% CI: 1.55 to 3.45 , 
A

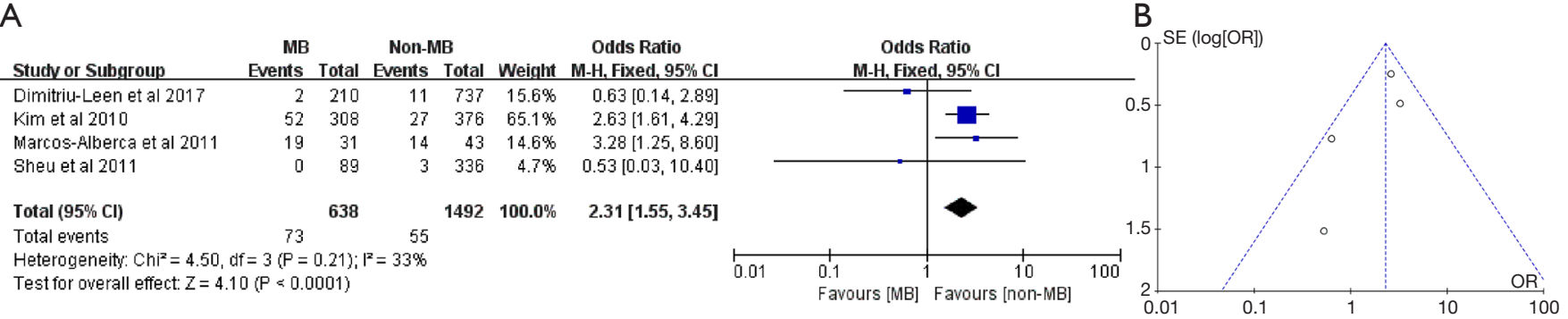

Figure 4 Pooled risk of angina requiring hospitalization. (A) Forest plot of included studies describing angina requiring hospitalization during follow-up. Subjects with myocardial bridging had higher risk of experiencing angina requiring hospitalization; (B) corresponding funnel plot of included studies. MB, myocardial bridge; CI, confidence interval; OR, odds ratio; SE, standard error.

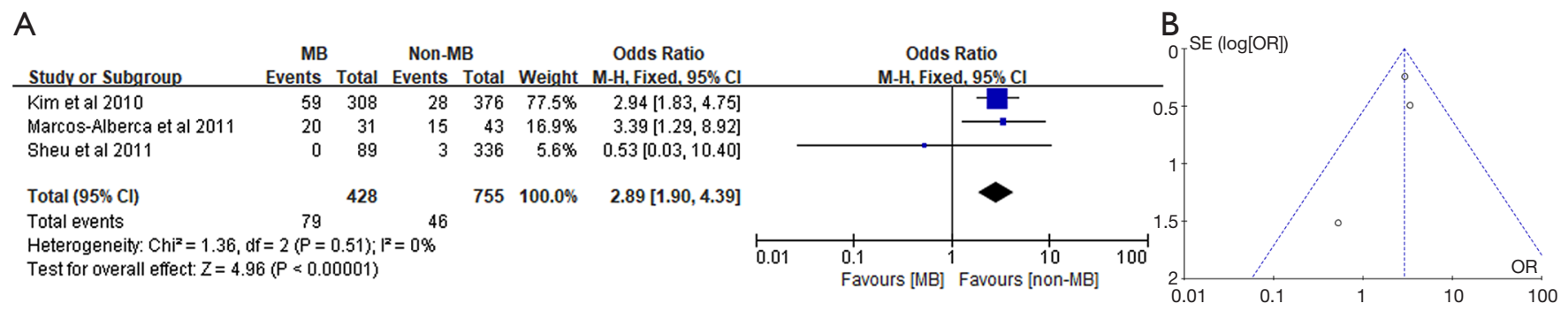

Figure 5 Pooled risk of the composite endpoint. (A) Forest plot of included studies describing the composite endpoint during followup. Subjects with myocardial bridging had higher risk of experiencing the composite endpoint. (B) Corresponding funnel plot of included studies. MB, myocardial bridge; CI, confidence interval; OR, odds ratio; SE, standard error.

$\mathrm{P}<0.0001)$ (Figure 4). There was no statistical heterogeneity between included studies (Cochrane $\mathrm{Q}=4.50, \mathrm{P}=0.21, \mathrm{I}^{2}$ $=33 \%$ ).

All-cause mortality was reported in three studies comprising a total of 46 events among 1,965 subjects $(11,15,18)$. The pooled incidences of all-cause mortality were $1.7 \%$ and $2.6 \%$ in subjects with $\mathrm{MB}$ and without $\mathrm{MB}$, respectively. Subjects with $\mathrm{MB}$ had no significant increase in the risk of all-cause mortality compared with subjects without MB (OR: 0.75 ; 95\% CI: 0.38 to $1.49, \mathrm{P}=0.41$ ) (Figure S2). There was no statistical heterogeneity between included studies (Cochrane $\mathrm{Q}=1.90, \mathrm{P}=0.39, \mathrm{I}^{2}=0 \%$ ).

\section{Composite endpoint}

Of six included studies, three studies involving 1,183 subjects, reported composite endpoint comprising of ACEs, non-cardiac death and angina requiring hospitalization (16-18). The pooled incidences of the composite endpoint were $18.5 \%$ and $6.1 \%$ in subjects with $M B$ and without $M B$, respectively. Subjects with $M B$ had an increased risk of experiencing the composite endpoint compared with subjects without MB (OR: 2.89; 95\% CI: 1.90 to 4.39, $\mathrm{P}<0.00001$ ) (Figure 5). There was no statistical heterogeneity between included studies (Cochrane $\mathrm{Q}=1.36$, $\left.\mathrm{P}=0.51, \mathrm{I}^{2}=0 \%\right)$.

\section{Discussion}

The present meta-analysis aims to examine the impact of $\mathrm{MB}$ on clinical prognosis in the general population which includes the latest cohort studies to date as far as we know. Our results indicate that $\mathrm{MB}$ is associated with an increased risk of ACEs and non-fatal MI in the present study. Thus, our findings may have important implications with regard to clinical practice and may alter our previous conceptions and strategies to provide more attention and optimal management of $\mathrm{MB}$.

The pooled prevalence of $\mathrm{MB}$ with $30.5 \%$ in the present study is similar to that in the prospective study by Rubinshtein et al. and the average prevalence of $25 \%$ detected in autopsy which is usually regarded as a reference 
standard $(7,11,16)$. Generally, according to previous studies, depiction rate of MB in coronary angiography, coronary computed tomographic angiography and autopsy is increased in ascending order $(4,5,7,11)$. The prevalence of $\mathrm{MB}$ on coronary computed tomographic angiography in more recent studies is in accordance with autopsy series, which may be attributed to the increasingly high spatial resolution of newer generation computed tomography capable of refining $M B(4,19)$.

Our key findings suggest that $\mathrm{MB}$ confers an increased risk of ACEs (OR: 1.71; 95\% CI: 1.29 to 2.26, $\mathrm{P}=0.0002$ ) and non-fatal MI (OR: 3.17; 95\% CI: 1.21 to $8.31, \mathrm{P}=0.02$ ) in subjects with $\mathrm{MB}$ compared with subjects without $\mathrm{MB}$, respectively. Thus, our findings are contrary to previous studies and traditional consideration that $\mathrm{MB}$ is a normal variant or a benign coronary anomaly $(11,15)$. Regarding clinical symptom, subjects with MB had an increased risk of angina requiring hospitalization (OR: $2.31 ; 95 \% \mathrm{CI}: 1.55$ to $3.45, \mathrm{P}<0.0001)$ compared with subjects without $\mathrm{MB}$.

There are several potential mechanisms that may attribute to the association of $\mathrm{MB}$ with ACEs or myocardial ischemia. First, MB itself mostly involves the LAD which is one of the most important coronary arteries and whose lesion commonly contributes to most MI or myocardial ischemia in obstructive coronary artery disease. Hemodynamic relevance of MB differs significantly with regard to its anatomy especially depth $(1,20)$. Second, multiple studies on MB using intracoronary ultrasound, Doppler and quantitative coronary angiography have revealed that systolic compression of $\mathrm{MB}$ persists into diastolic phase of cardiac cycle rather than that $\mathrm{MB}$ is just a systolic event (21-25). This finding is deemed highly unique as it can only be detected in the segment of $\mathrm{MB}$ with systolic compression $(1,26)$. Moreover, findings by intracoronary Doppler demonstrate that $\mathrm{MB}$ compression delays luminal recovery in early diastole which may impair diastolic hemodynamics, which is left unidentified before (21). Additionally, the degree of the systolic compression of $\mathrm{MB}$ is positively associated with reduction of luminal diameter and corresponding decrease in flow and flow reserve during diastole (27). Third, previous studies reveal endothelial dysfunction of the tunneled coronary artery beneath MB $(28,29)$. Furthermore, reduced expression of some vasoactive agents like endothelial nitric oxide synthase, endothelin-1, and angiotensinconverting enzyme at the $\mathrm{MB}$ site were ascertained to attribute to endothelial dysfunction of the tunneled coronary artery, which may predispose tunneled coronary artery to spasm at the same time $(4,28,29)$. Fourth, several studies demonstrated a higher incidence of cardiac death and nonfatal MI in subsets of patients with coronary artery spasm and without obstructive coronary artery disease $(30,31)$. Fifth, it has been found that vessel segment proximal to MB predisposes to development of atherosclerosis or formation of plaques, though vessel segment within $\mathrm{MB}$ is protected from development of atherosclerotic lesions $(6,10,32)$. Disturbed retrograde flow produced by systolic compression of $\mathrm{MB}$ alters significantly shear stress on the coronary artery wall proximal to $\mathrm{MB}$ leading to atherosclerosis of corresponding part of the coronary artery $(6,32)$. This finding has been thought to increase the risk of ACEs or myocardial ischemia.

\section{Limitations}

This meta-analysis has some limitations. First, our study itself is prone to inherent limitations of this kind of analysis like publication bias. Our data are confined to rely on published studies. Second, most included studies are retrospective except the study by Rubinshtein and colleagues (11). Therefore, our study may have limitations, potential confounding and biases of all retrospective studies. However, prospectively observational study examining the impact of MB on prognosis is relatively lacking, especially with a comparison group of subjects without $\mathrm{MB}$, and has relatively small sample size. Third, of six included studies, the study by Kim et al. uses coronary angiography to detect $\mathrm{MB}$, which differs from the other five included studies with coronary computed tomographic angiography used and is usually thought to has a lower detection rate of MB. However, Kim et al. administered intracoronary nitroglycerin in order to well define $\mathrm{MB}$ once suspecting MB during coronary angiography (18). Besides, six included studies only provided limited information about functional effects of $\mathrm{MB}$ or clinical symptoms in participants. Fourth, tools employed for diagnosis of MB may be different among included studies in different periods. However, the time span is relatively short, so differences in terms of anatomical definition and functional relapse are slight. Fifth, followup duration in two included studies was relatively short and none of included studies provided information on loss to follow-up, which may add some bias to our study $(16,17)$. Sixth, our study could not respectively refine association of different MB types with presence/magnitude of coronary mal-perfusion and prognosis basing on current evidences due to a lack of source data. 


\section{Conclusions}

$\mathrm{MB}$ is not uncommon especially assessed on coronary computed tomographic angiography. Subjects with MB and without obstructive coronary artery disease have increased risk of experiencing ACEs including cardiac death and nonfatal MI, as well as angina requiring hospitalization. These findings may have substantially important implication which may alter our traditional conception of $\mathrm{MB}$ as well as clinical practice. However, the present finding needs further prospectively longitudinal multicenter study with large sample size to validate.

\section{Acknowledgments}

Funding: This work was supported by National Natural Science Foundation of China (No. 81570276) and Beijing Science and Technology Program of China (No. Z161100000516154).

\section{Footnote}

Conflicts of Interest: The authors have no conflicts of interest to declare.

Ethical Statement: The authors are accountable for all aspects of the work in ensuring that questions related to the accuracy or integrity of any part of the work are appropriately investigated and resolved.

Open Access Statement: This is an Open Access article distributed in accordance with the Creative Commons Attribution-NonCommercial-NoDerivs 4.0 International License (CC BY-NC-ND 4.0), which permits the noncommercial replication and distribution of the article with the strict proviso that no changes or edits are made and the original work is properly cited (including links to both the formal publication through the relevant DOI and the license). See: https://creativecommons.org/licenses/by-nc-nd/4.0/.

\section{References}

1. Tarantini G, Migliore F, Cademartiri F, et al. Left anterior descending artery myocardial bridging: A clinical approach. J Am Coll Cardiol 2016;68:2887-99.

2. Corban MT, Hung OY, Eshtehardi P, et al. Myocardial bridging: Contemporary understanding of pathophysiology with implications for diagnostic and therapeutic strategies.
J Am Coll Cardiol 2014;63:2346-55.

3. Alegria JR, Herrmann J, Holmes DR, Jr., et al. Myocardial bridging. Eur Heart J 2005;26:1159-68.

4. Lee MS, Chen CH. Myocardial Bridging: An Up-to-Date Review. J Invasive Cardiol 2015;27:521-8.

5. Rogers IS, Tremmel JA, Schnittger I. Myocardial bridges: Overview of diagnosis and management. Congenit Heart Dis 2017;12:619-23.

6. Ishikawa Y, Akasaka Y, Suzuki K, et al. Anatomic properties of myocardial bridge predisposing to myocardial infarction. Circulation 2009;120:376-83.

7. Möhlenkamp S. Update on myocardial bridging. Circulation 2002;106:2616-22.

8. Rossi L, Dander B, Nidasio GP, et al. Myocardial bridges and ischemic heart disease. Eur Heart J 1980;1:239-45.

9. Geiringer E. The mural coronary. Am Heart J 1951;41:359-68.

10. Uusitalo V, Saraste A, Pietilä M, et al. The Functional Effects of Intramural Course of Coronary Arteries and its Relation to Coronary Atherosclerosis. JACC Cardiovasc Imaging 2015;8:697-704.

11. Rubinshtein R, Gaspar T, Lewis BS, et al. Long-term prognosis and outcome in patients with a chest pain syndrome and myocardial bridging: a 64-slice coronary computed tomography angiography study. Eur Heart J Cardiovasc Imaging 2013;14:579-85.

12. Wells GA, Shea B, O'Connell O, et al. The newcastleottawa scale (nos) for assessing the quality of nonrandomised studies in meta-analyses (25 january 2017). 2017. Available online: http://www.ohri.ca/programs/ clinical_epidemiology/oxford.asp

13. Kunutsor SK, Seidu S, Khunti K. Statins and secondary prevention of venous thromboembolism: Pooled analysis of published observational cohort studies. Eur Heart J 2017;38:1608-12.

14. Liu G, Qu Y, Chen X, et al. Measurements of myocardial bridges on computed tomography predict presence of clinical symptoms and outcomes of adverse heart events: A retrospective study in a large population from china. Acta Radiol 2017;58:1068-76.

15. Dimitriu-Leen AC, van Rosendael AR, Smit JM, et al. Long-Term Prognosis of Patients With Intramural Course of Coronary Arteries Assessed With CT Angiography. JACC Cardiovasc Imaging 2017;10:1451-8.

16. Sheu MH, Chen YD, Kuo YS, et al. Myocardial bridging in taiwanese: Noninvasive assessment by 64-detector row coronary computed tomographic angiography. J Chin Med Assoc 2011;74:164-8. 
17. Marcos-Alberca P, Goncalves A, Golfin CF, et al. Clinical outcomes of patients with intramyocardial bridging diagnosed by multi-detector cardiac computed tomography. Int J Cardiol 2011;148:123-5.

18. Kim SS, Jeong MH, Kim HK, et al. Long-term clinical course of patients with isolated myocardial bridge. Circ J 2010;74:538-43.

19. Konen E, Goitein O, Sternik L, et al. The prevalence and anatomical patterns of intramuscular coronary arteries: A coronary computed tomography angiographic study. J Am Coll Cardiol 2007;49:587-93.

20. Leschka S, Koepfli P, Husmann L, et al. Myocardial bridging: Depiction rate and morphology at ct coronary angiography--comparison with conventional coronary angiography. Radiology 2008;246:754-62.

21. Escaned J, Cortés J, Flores A, et al. Importance of diastolic fractional flow reserve and dobutamine challenge in physiologic assessment of myocardial bridging. J Am Coll Cardiol 2003;42:226-33.

22. Ge J, Jeremias A, Rupp A, et al. New signs characteristic of myocardial bridging demonstrated by intracoronary ultrasound and doppler. Eur Heart J 1999;20:1707-16.

23. Tio RA, Van Gelder IC, Boonstra PW, et al. Myocardial bridging in a survivor of sudden cardiac near-death: role of intracoronary doppler flow measurements and angiography during dobutamine stress in the clinical evaluation. Heart 1997;77:280-2.

24. Klues HG, Schwarz ER, vom Dahl J, et al. Disturbed intracoronary hemodynamics in myocardial bridging: Early normalization by intracoronary stent placement. Circulation 1997;96:2905-13.

Cite this article as: Zhu C, Wang S, Cui H, Tang B, Wang S. Associations of myocardial bridging with adverse cardiac events: a meta-analysis of published observational cohort studies involving 4,556 individuals. Ann Transl Med 2020;8(6):369. doi: 10.21037/atm.2020.02.24
25. Aleksandric S, Djordjevic-Dikic A, Beleslin B, et al. Noninvasive assessment of myocardial bridging by coronary flow velocity reserve with transthoracic doppler echocardiography: Vasodilator vs. Inotropic stimulation. Int J Cardiol 2016;225:37-45.

26. Ge J, Erbel R, Rupprecht HJ, et al. Comparison of intravascular ultrasound and angiography in the assessment of myocardial bridging. Circulation 1994;89:1725-32.

27. Schwarz ER, Klues HG, vom Dahl J, et al. Functional characteristics of myocardial bridging. A combined angiographic and intracoronary doppler flow study. Eur Heart J 1997;18:434-42.

28. Kim JW, Seo HS, Na JO, et al. Myocardial bridging is related to endothelial dysfunction but not to plaque as assessed by intracoronary ultrasound. Heart 2008;94:765-9.

29. Herrmann J, Higano ST, Lenon RJ, et al. Myocardial bridging is associated with alteration in coronary vasoreactivity. Eur Heart J 2004;25:2134-42.

30. Wang CH, Kuo LT, Hung MJ, et al. Coronary vasospasm as a possible cause of elevated cardiac troponin I in patients with acute coronary syndrome and insignificant coronary artery disease. Am Heart J 2002;144:275-81.

31. Bory M, Pierron F, Panagides D, et al. Coronary artery spasm in patients with normal or near normal coronary arteries. Long-term follow-up of 277 patients. Eur Heart J 1996;17:1015-21.

32. Ishikawa Y, Akasaka Y, Ito K, et al. Significance of anatomical properties of myocardial bridge on atherosclerosis evolution in the left anterior descending coronary artery. Atherosclerosis 2006;186:380-9. 
Table S1 PRISMA checklist*

\begin{tabular}{|c|c|c|c|}
\hline Section/topic & \# & Checklist item & Reported on page ${ }^{\#}$ \\
\hline \multicolumn{4}{|l|}{ Title } \\
\hline Title & 1 & Identify the report as a systematic review, meta-analysis, or both & 1 \\
\hline \multicolumn{4}{|l|}{ Abstract } \\
\hline Structured summary & 2 & $\begin{array}{l}\text { Provide a structured summary including, as applicable: background; objectives; data sources; study eligibility criteria, participants, and interventions; study appraisal } \\
\text { and synthesis methods; results; limitations; conclusions and implications of key findings; systematic review registration number }\end{array}$ & 1 \\
\hline \multicolumn{4}{|l|}{ Introduction } \\
\hline Rationale & 3 & Describe the rationale for the review in the context of what is already known & $1-2$ \\
\hline \multicolumn{4}{|l|}{ Methods } \\
\hline Protocol and registration & 5 & Indicate if a review protocol exists, if and where it can be accessed (e.g., Web address), and, if available, provide registration information including registration number & None \\
\hline Eligibility criteria & 6 & $\begin{array}{l}\text { Specify study characteristics (e.g., PICOS, length of follow-up) and report characteristics (e.g., years considered, language, publication status) used as criteria for eligibility, } \\
\text { giving rationale }\end{array}$ & Methods \\
\hline Information sources & 7 & Describe all information sources (e.g., databases with dates of coverage, contact with study authors to identify additional studies) in the search and date last searched & Methods \\
\hline Search & 8 & Present full electronic search strategy for at least one database, including any limits used, such that it could be repeated & Table S2 \\
\hline Study selection & 9 & State the process for selecting studies (i.e., screening, eligibility, included in systematic review, and, if applicable, included in the meta-analysis) & Methods \\
\hline Data items & 11 & List and define all variables for which data were sought (e.g., PICOS, funding sources) and any assumptions and simplifications made & Methods \\
\hline Risk of bias in individual studies & 12 & $\begin{array}{l}\text { Describe methods used for assessing risk of bias of individual studies (including specification of whether this was done at the study or outcome level), and how this information } \\
\text { is to be used in any data synthesis }\end{array}$ & Methods \\
\hline Summary measures & 13 & State the principal summary measures (e.g., risk ratio, difference in means) & Methods \\
\hline Synthesis of results & 14 & Describe the methods of handling data and combining results of studies, if done, including measures of consistency (e.g., $\left.I^{2}\right)$ for each meta-analysis & Methods \\
\hline Risk of bias across studies & 15 & Specify any assessment of risk of bias that may affect the cumulative evidence (e.g., publication bias, selective reporting within studies) & Methods \\
\hline Additional analyses & 16 & Describe methods of additional analyses (e.g., sensitivity or subgroup analyses, meta-regression), if done, indicating which were pre-specified & None \\
\hline \multicolumn{4}{|l|}{ Results } \\
\hline Study selection & 17 & Give numbers of studies screened, assessed for eligibility, and included in the review, with reasons for exclusions at each stage, ideally with a flow diagram & Figure 1 \\
\hline Study characteristics & 18 & For each study, present characteristics for which data were extracted (e.g., study size, PICOS, follow-up period) and provide the citations & Tables 1,2 \\
\hline Risk of bias within studies & 19 & Present data on risk of bias of each study and, if available, any outcome-level assessment (see Item 12) & Table S3 \\
\hline Results of individual studies & 20 & $\begin{array}{l}\text { For all outcomes considered (benefits or harms), present, for each study: (I) simple summary data for each intervention group and (II) effect estimates and confidence } \\
\text { intervals, ideally with a forest plot }\end{array}$ & Results and Figures 2,5 \\
\hline Synthesis of results & 21 & Present results of each meta-analysis done, including confidence intervals and measures of consistency & Results and Figures 2-5 \\
\hline Risk of bias across studies & 22 & Present results of any assessment of risk of bias across studies (see Item 15) & Results, Figure 3, and Table S3 \\
\hline Additional analysis & 23 & Give results of additional analyses, if done (e.g., sensitivity or subgroup analyses, meta-regression) (see Item 16) & None \\
\hline Limitations & 25 & Discuss limitations at study and outcome level (e.g., risk of bias), and at review level (e.g., incomplete retrieval of identified research, reporting bias) & Limitations \\
\hline Conclusions & 26 & Provide a general interpretation of the results in the context of other evidence, and implications for future research & Conclusions \\
\hline \multicolumn{4}{|l|}{ Funding } \\
\hline Funding & 27 & Describe sources of funding for the systematic review and other support (e.g., supply of data); role of funders for the systematic review & Funding \\
\hline
\end{tabular}


Table S2 Search strategy used in the PubMed database from 1960 to 31 March 2018

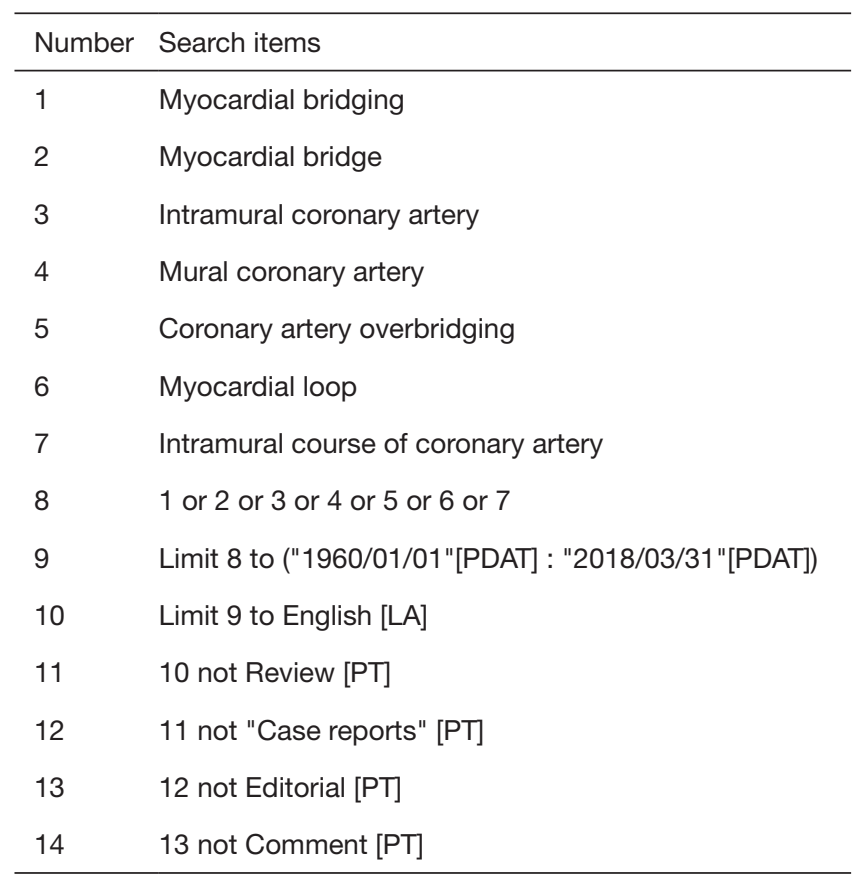

Table S3 Quality evaluation of included studies

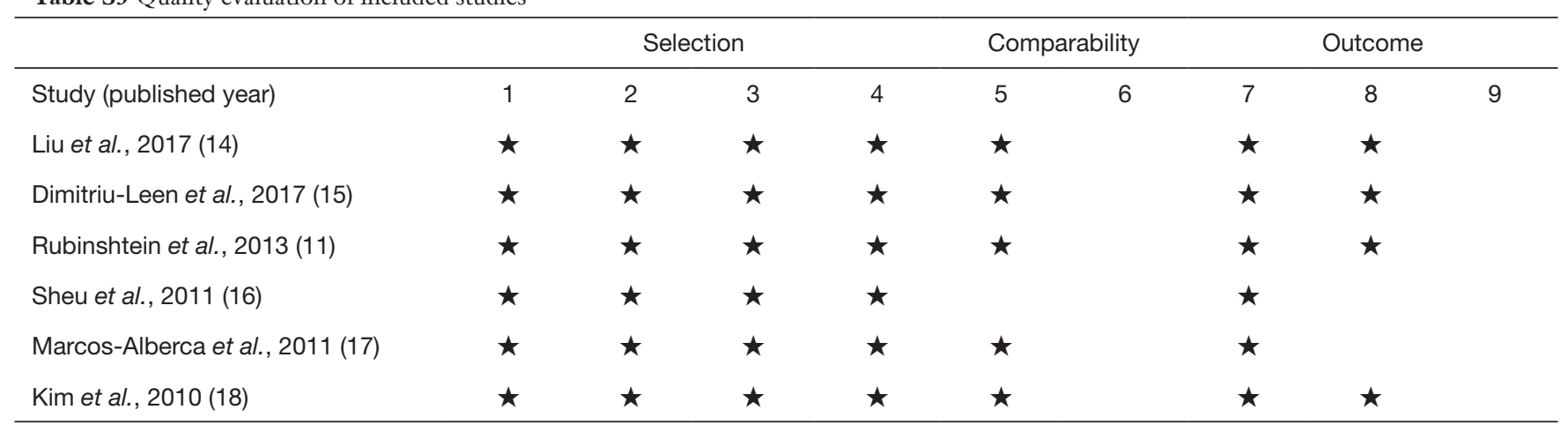


Table S4 MOOSE checklist*

\begin{tabular}{|c|c|}
\hline Checklist item & Brief description \\
\hline \multicolumn{2}{|l|}{ Reporting of background } \\
\hline Problem definition & $\begin{array}{l}\text { Data derived from small series have demonstrated an association of myocardial bridge (MB) with adverse cardiac events, while MB has been } \\
\text { traditionally considered as a benign condition. Hence, the precise clinical implications of MB on prognosis remains inconsistent }\end{array}$ \\
\hline Hypothesis statement & MB may have an association with adverse cardiac events (ACEs) \\
\hline Description of study outcomes & $\begin{array}{l}\text { ACEs including cardiovascular death and non-fatal myocardial infarction (MI); secondary outcomes like non-fatal MI, angina requiring hospitalization, and all- } \\
\text { cause mortality; composite endpoint defined as a combination of ACEs, non-cardiac death and angina requiring hospitalization }\end{array}$ \\
\hline Type of exposure & With MB \\
\hline Type of study designs used & Population-based cohort studies \\
\hline Study population & Populations referred for computed tomographic coronary angiography or coronary angiography in hospital \\
\hline \multicolumn{2}{|l|}{ Reporting of search strategy should include } \\
\hline Qualifications of searchers & Changsheng Zhu, MD; Shuiyun Wang, MD \\
\hline \multirow[t]{2}{*}{ Search strategy, including time period included in the synthesis and keywords } & Time period: from inception of PubMed to March 31, 2018 \\
\hline & Search strategy: Table $S 2$ \\
\hline Databases and registries searched & PubMed \\
\hline Search software used, name and version, including special features & Endnote $X 8.2$ was used to manage references \\
\hline Use of hand searching & Additional reference lists of relevant articles were searched \\
\hline List of citations located and those excluded, including justifications & Details of the literature search process are presented in the flow chart (Figure 1). List of excluded citations is available on request \\
\hline Method of addressing articles published in languages other than English & The search was restricted to the English language \\
\hline Method of handling abstracts and unpublished studies & None \\
\hline Description of any contact with authors & Not applicable \\
\hline \multicolumn{2}{|l|}{ Reporting of methods should include } \\
\hline $\begin{array}{l}\text { Description of relevance or appropriateness of studies assembled for assessing the } \\
\text { hypothesis to be tested }\end{array}$ & Methods section \\
\hline Rationale for the selection and coding of data & Extracted data from included studies were related to population characteristics, study design, exposure and outcome measurements \\
\hline Assessment of confounding & Not applicable \\
\hline $\begin{array}{l}\text { Assessment of study quality, including blinding of quality assessors; stratification or } \\
\text { regression on possible predictors of study results }\end{array}$ & $\begin{array}{l}\text { Study quality was assessed with the nine-star Newcastle-Ottawa Scale (NOS) which is pre-defined criteria including population representativeness, compara- } \\
\text { bility, ascertainment of outcome (Table S3) }\end{array}$ \\
\hline Assessment of heterogeneity & Heterogeneity of the studies was evaluated with $I^{2}$ statistic \\
\hline Description of statistical methods in sufficient detail to be replicated & Details of statistical methods were described in the Methods section \\
\hline Provision of appropriate tables and graphics & Tables 1,2, Figures 1-5 \\
\hline \multicolumn{2}{|l|}{ Reporting of results should include } \\
\hline Graph summarizing individual study estimates and overall estimate & Figures 2-5 \\
\hline Table giving descriptive information for each study included & Tables 1,2 \\
\hline Results of sensitivity testing & Not applicable \\
\hline Indication of statistical uncertainty of findings & $95 \%$ confidence intervals were calculated for all summary estimates \\
\hline \multicolumn{2}{|l|}{ Reporting of discussion should include } \\
\hline Quantitative assessment of bias & Publication bias was assessed with funnel plot \\
\hline Justification for exclusion & All studies were excluded based on the pre-defined inclusion and exclusion criteria in the Methods section \\
\hline Assessment of quality of included studies & Quality assessment of included studies was described in Methods section \\
\hline \multicolumn{2}{|l|}{ Reporting of conclusions should include } \\
\hline Consideration of alternative explanations for observed results & Discussion section \\
\hline Generalization of the conclusions & Results section \\
\hline Guidelines for future research & Further prospective multicentre studies with large sample size are needed to confirm current findings \\
\hline Disclosure of funding source & Dr. Shuiyun Wang has received grants from National Natural Science Foundation of China \\
\hline
\end{tabular}

*, Stroup DF, Berlin JA, Morton SC, et al. Meta-analysis of observational studies in epidemiology: a proposal for reporting. Meta-analysis Of Observational Studies in Epidemiology (MOOSE) group. JAMA 2000;283:2008-12. 


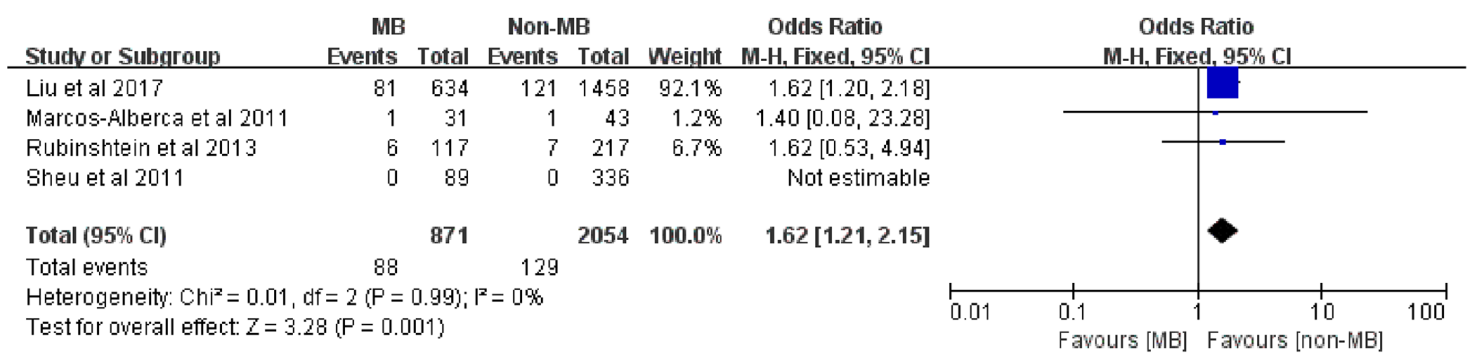

Figure S1 Sensitivity analysis only including five studies which used coronary computed tomographic angiography for detection of myocardial bridging. Subjects with myocardial bridging had higher risk of experiencing adverse cardiac events. MB, myocardial bridge; CI, confidence interval.

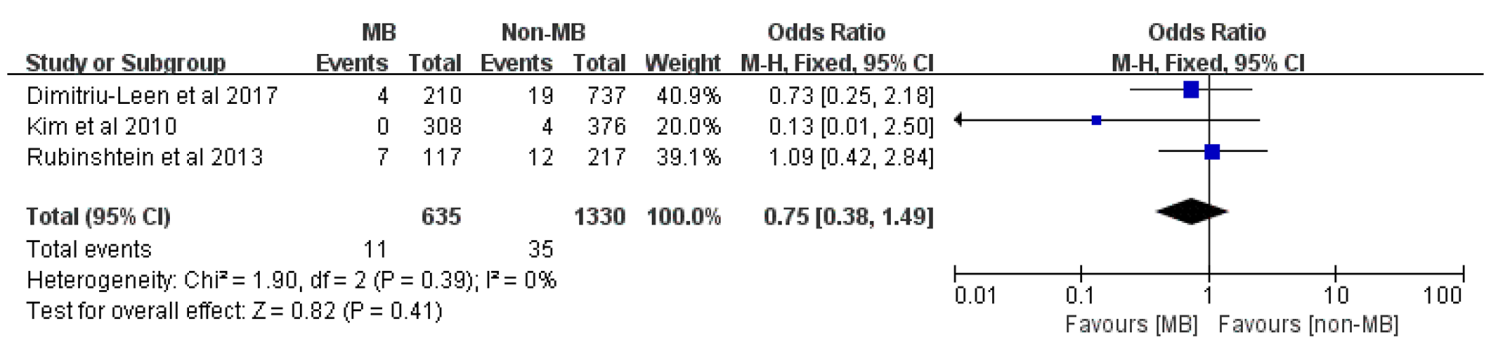

Figure S2 Forest plot of included studies describing all-cause mortality during follow-up. Subjects with myocardial bridging had higher risk of experiencing all-cause mortality. MB, myocardial bridge; CI, confidence interval. 